\title{
A new era at attention, perception, \& psychophysics
}

Starting with this issue, Attention, Perception, \& Psychophysics and, indeed, all of the Psychomonic Society journals, will be published by Springer. For all of its previous history, AP\&P has been published by the Publications Office of the Psychonomic Society. We are deeply grateful to the Publications Office for all their labors over the years and we are pleased that a number of people who we have worked with over the years will continue working on AP\&P for Springer.

We are excited by Springer's abilities to help this journal thrive in a publishing world that is increasingly electronic and increasingly global. AP\&P will retain its commitment to publish the best research in our field, but now that research will reach its intended audience faster. Within a very few weeks after your paper is accepted, it will be published, in citable form, in the OnlineFirst section of SpringerLink. If you are a member of the Psychonomic Society or if you have access to the journal in another way, you will be able to see and download all of the latest content from the journal. There will be more color in the figures and easier access to supplementary materials. All of the earlier volumes will be openly accessible from SpringerLink.

Because of Springer's global reach, we expect AP\&P to be available in a wider range of libraries around the world. This will increase the readership and the impact of the work you publish here. We on the AP\&P Editorial Board reaffirm our commitment to making the review process fair, thorough, and expeditious. We are eager to hear your opinions and suggestions for the journal. Feel free to send comments towolfe@search.bwh.harvard.edu.

Speaking of email, you can (and should!) sign up for regular electronic delivery of the table of contents at http://www. springer.com/psychology/cognitive+psychology/journal/13414

\section{Jeremy Wolfe}

Editor 